\title{
Enhancing the Performance of Germanium Channel nMOSFET Using Phosphorus Dopant Segregation
}

\author{
Che-Wei Chen, Ju-Yuan Tzeng, Cheng-Ting Chung, Hung-Pin Chien, Chao-Hsin Chien, \\ Guang-Li Luo, Pei-Yu Wang, and Bing-Yue Tsui
}

\begin{abstract}
In this letter, we present a high performance Ge n-channel metal-oxide-semiconductor field-effect transistor (MOSFET) with a NiGe Schottky junction source/drain fabricated using phosphorus dopant segregation. Phosphorus atoms were implanted into $\mathrm{NiGe}$ and then driven toward the NiGe/p-Ge interface to depin the Fermi level and form a Schottky junction. A high effective barrier height $\left(\Phi_{B p}\right)$ of $0.57 \mathrm{eV}$, resulting in a high junction current ratio of $>10^{4}$ at the applied voltage $\left|V_{\mathrm{a}}\right|= \pm 1 \mathrm{~V}$. The nMOSFET exhibited a high $I_{\text {ON }} / I_{\text {OFF }}$ ratio of $\sim 8 \times 10^{3}\left(I_{D}\right), \sim 10^{5}\left(I_{S}\right)$, and a subthreshold swing of $138 \mathrm{mV} /$ decade. The nMOSFET developed in this letter exhibited greater transconductance and a drain leakage current that is more than two orders of magnitude lower compared with nMOSFETs with the conventional $n^{+/ p}$ junction.
\end{abstract}

Index Terms-Shottky barrier height, dopant segregation, NiGe, nMOSFET.

\section{INTRODUCTION}

G ERMANIUM (Ge) channel devices have been regarded as a replacement for $\mathrm{Si}$ channel metaloxide-semiconductor field-effect transistors (MOSFETs) because Ge has a high carrier mobility [1], [2]. Although the high performance Ge p-channel MOSFETs has been demonstrated [3], [4], fabricating high performance $\mathrm{Ge}$ n-channel MOSFETs remains extremely challenging because n-type dopants diffuse rapidly and have relatively low solid solubility [5], [6]. Moreover, n-type dopants, even with a high activation temperature, have been reported to exhibit a poor activation efficiency [7]-[9], making the formation of a S/D with adequately low resistance very difficult. A possible solution is to use a Schottky junction S/D

Manuscript received October 1, 2013; revised November 4, 2013 and November 14, 2013; accepted November 15, 2013. Date of publication December 6, 2013; date of current version December 20, 2013. This work was supported in part by the National Science Council of Taiwan under Grant NSC 101-2628-E-009-011-MY3 and in part by the NCTU-UCB I-RiCE Program under Grant NSC-102-2911-I-009-302. The review of this letter was arranged by Editor J. Cai.

C.-W. Chen, J.-Y. Tzeng, C.-T. Chung, H.-P. Chien, P.-Y. Wang, and B.-Y. Tsui are with the Department of Electronics Engineering and the Institute of Electronics, National Chiao Tung University, Hsinchu 30010, Taiwan.

C.-H. Chien is with the Department of Electronics Engineering and the Institute of Electronics, National Chiao Tung University, Hsinchu 30010, Taiwan, and also with the National Nano Device Laboratories, Hsinchu 30078, Taiwan (e-mail: chchien@faculty.nctu.edu.tw).

G.-L. Luo is with the National Nano Device Laboratories, Hsinchu 30078, Taiwan.

Color versions of one or more of the figures in this letter are available online at http://ieeexplore.ieee.org.

Digital Object Identifier 10.1109/LED.2013.2291774 (or metal S/D) to replace the conventional $\mathrm{n}^{+} / \mathrm{p}$ junction S/D. By using a phosphorus dopant segregation (DS) technique, the $\mathrm{NiGe/p}-\mathrm{Ge}$ junction can be changed from an ohmic contact to a Schottky contact. Recently, many elements from group $\mathrm{V}$, including phosphorus, arsenic, and antimony, have been proven to modulate the Schottky barrier height $(\mathrm{SBH})$ of NiGe/Ge [10]-[13] because they segregate at the interface. Li et al. successfully fabricated a $\mathrm{NiGe} / \mathrm{p}-\mathrm{Ge}$ Schottky diode with a low electron SBH of $0.1 \mathrm{eV}$ by implanting phosphorus after NiGe formation [12]. Koike et al. induced phosphorus atoms in the NiGe/p-Ge to tune the SBH [13]. Even though the underlying mechanism for the modulation of the SBH value has been intensively discussed [10]-[13], the fabrication of high performance nMOSFETs with a Schottky junction S/D by using a DS technique has not yet been demonstrated in the literatures.

In this letter, a high performance Ge nMOSFETs with a NiGe/p-Ge Schottky junction S/D was fabricated using a phosphorus DS technique. The resultant high effective barrier height $\left(\Phi_{\mathrm{Bp}}\right)$ led to a considerably low leakage current in the devices. High $I_{\mathrm{ON}} / I_{\mathrm{OFF}}$ ratio and good SS were achieved. Therefore, using this approach greatly improved the electrical characteristics of Ge channel nMOSFETs.

\section{DEVICE FABRICATION}

A $400 \mathrm{~nm}$ plasma enhanced chemical vapor deposition (PECVD) isolation oxide was deposited on a p-Ge (100) substrate with a resistivity of $0.1-0.6 \mathrm{ohm}-\mathrm{cm}$ after cleaning. The S/D pattern was defined by using photolithography, and the subsequent oxide etch was accomplished by using a buffer oxide etchant. Nickel was deposited by sputtering, and a liftoff process was performed; rapid thermal annealing (RTA) at $200{ }^{\circ} \mathrm{C}$ for $60 \mathrm{~s}$ was used to form the NiGe alloy as the metal S/D of the nMOSFET. The phosphorus dopants $\left(10 \mathrm{keV}, 1 \times 10^{15} \mathrm{~cm}^{-2}\right)$ were then implanted in the metal S/D regions. The dopants segregated at the $\mathrm{NiGe} / \mathrm{p}-\mathrm{Ge}$ interface when they were activated using RTA at $500{ }^{\circ} \mathrm{C}$ for $60 \mathrm{~s}$. Moreover, an interesting approach often referred to as the snowplow effect, or the pile-up of phosphorus dopants, has been verified by using a secondary ion mass spectrometry (SIMS) depth profile [10]-[12], [14]. $\mathrm{A} \mathrm{GeO}_{2}$ was used as a surface passivation layer using rapid thermal oxidation (RTO) at $520{ }^{\circ} \mathrm{C}$ for $30 \mathrm{~s}$ and then an $\mathrm{Al}_{2} \mathrm{O}_{3}$ deposition was performed by atomic layer deposition (ALD) after the active area was defined. Finally, the $\mathrm{Al}(400 \mathrm{~nm})$ gate and the metallization contact were established by using an evaporator. 

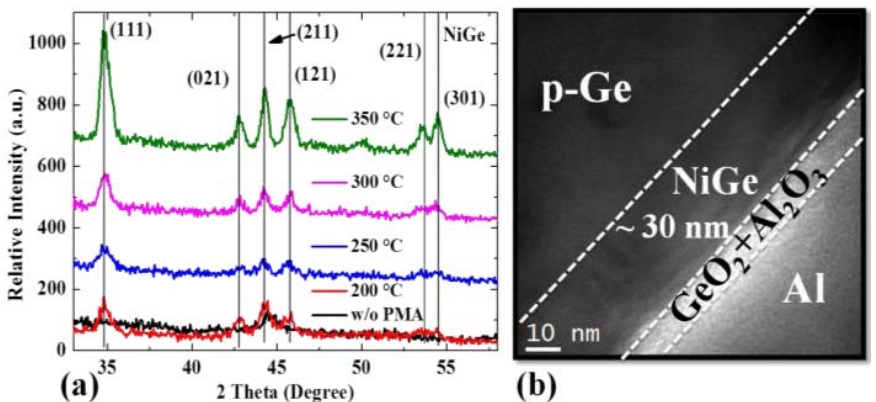

(b)

Fig. 1. (a) GIXRD spectra of NiGe films with various annealing temperatures. (b) TEM picture of gate-to-drain overlapping region of nMOSFET device; gate stack and $\mathrm{NiGe} / \mathrm{p}-\mathrm{Ge}$ junction are shown.
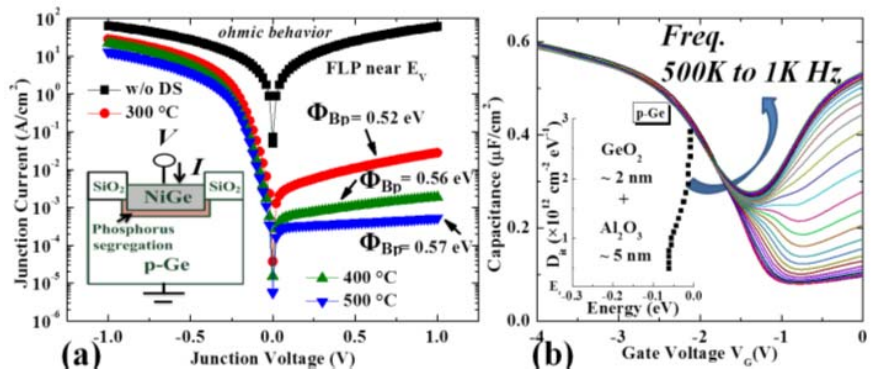

Fig. 2. (a) $I-V$ characteristics of $\mathrm{NiGe} / \mathrm{p}-\mathrm{Ge}$ diodes with/without phosphorus DS with different annealing temperatures. The inset is the schematic illustration of junction cross section. (b) $C-V$ characteristics of $\mathrm{Al} / \mathrm{Al}_{2} \mathrm{O}_{3} / \mathrm{GeO}_{2} / \mathrm{p}-\mathrm{Ge}$ structure and $D_{\text {it }}$ distribution near the Ge midgap (inset).

nMOSFETs with the conventional $\mathrm{n}^{+} / \mathrm{p}$ junction S/D were also accomplished for comparison.

\section{Results AND Discussion}

Fig. 1(a) shows the grazing incidence X-ray diffraction (GIXRD) spectra recorded at various annealing temperatures used for verifying NiGe formation. The peaks corresponding to (111), (021), (211), (121), (221), and (301) NiGe were clearly identified. Ni/n-Ge test Schottky junctions were also employed to determine the optimal PMA conditions (not shown). Performing RTA at $200{ }^{\circ} \mathrm{C}$ for $60 \mathrm{~s}$ was sufficient to form the $\mathrm{NiGe}$ phase. The transmission electron microscopy (TEM) image of the gate-to-drain overlapping region of the nMOSFET is shown in Fig. 1(b). The thickness of the NiGe film and that of the $\mathrm{GeO}_{2}+\mathrm{Al}_{2} \mathrm{O}_{3}$ were around $30 \mathrm{~nm}$ and $7 \mathrm{~nm}$, respectively; the device structure is shown in the inset of Fig. 3(a).

Fig. 2(a) shows the $I-V$ characteristics of the $\mathrm{NiGe} / \mathrm{p}-\mathrm{Ge}$ contacts fabricated using different annealing temperatures $\left(300{ }^{\circ} \mathrm{C}, 400{ }^{\circ} \mathrm{C}\right.$, and $\left.500{ }^{\circ} \mathrm{C}\right)$ with and without phosphorus DS. It was clear that the control sample without DS exhibited ohmic contact behavior caused by the strong Fermi level pinning (FLP) near the valence band and the resultant low $\Phi_{\mathrm{Bp}}$. Rectifying behavior was obtained by implanting phosphorus dopants into NiGe and subsequently conducting RTA. Phosphorus atoms are segregate near the $\mathrm{NiGe} / \mathrm{p}-\mathrm{Ge}$ interface when using RTA. The highest value of the effective $\Phi_{\mathrm{Bp}}$ extracted from the $I-V$ characteristics [15] was $\sim 0.57 \mathrm{eV}$, which was
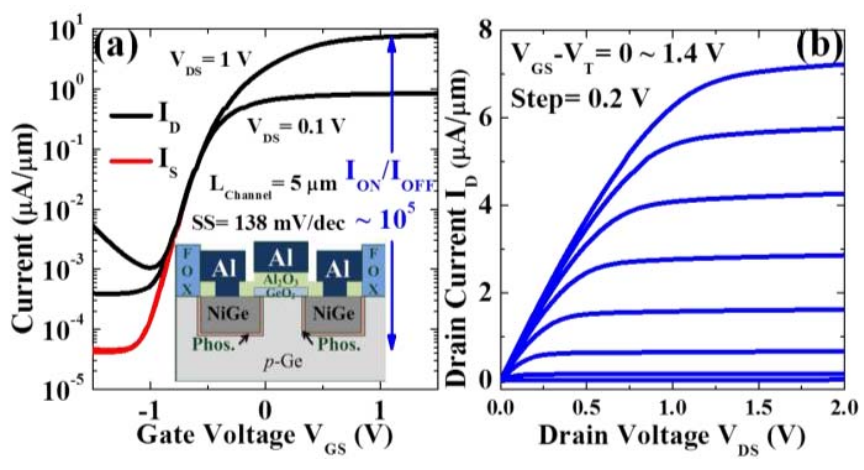

Fig. 3. (a) Transfer characteristics of Ge nMOSFET with a NiGe Schottky junction $\mathrm{S} / \mathrm{D}$ and the $I_{\mathrm{ON}} / I_{\mathrm{OFF}}$ ratio is of $\sim 10^{5}\left(I_{\mathrm{S}}\right)$. Schematic diagram of device structure (inset). (b) $I_{\mathrm{D}}-V_{\mathrm{DS}}$ output characteristic of device and the saturation current is of $4 \mu \mathrm{A} / \mu \mathrm{m}$ at $V_{\mathrm{DS}}=1 \mathrm{~V}$ and $V_{\mathrm{GS}}-V_{\mathrm{T}}=1 \mathrm{~V}$.

beneficial in restraining the reverse leakage current of the Schottky junction. Fig. 2(b) shows the $C-V$ characteristics of metal-oxide-semiconductor (MOS) capacitor used to examine the surface interface state density $\left(D_{\mathrm{it}}\right)$ of the $\mathrm{Al}_{2} \mathrm{O}_{3} / \mathrm{GeO}_{2} /$ p-Ge stack; the inset shows the extracted $D_{\text {it }}$ distribution near the Ge midgap obtained using a conductance method at room temperature [15]. Based on analyzing the $C-V$ curves, a small hump was observed in the weak inversion as the measuring frequency decreased. The voltage for the hump corresponded to that at which the peak conductance of the G-V curves (not shown) happens; the peak conductance arose from generationrecombination through $D_{\text {it }}[16]$. The estimated $D_{\text {it }}$ value is of $\sim 3 \times 10^{12} \mathrm{~cm}^{-2} \mathrm{eV}^{-1}$ near the Ge mid-gap and the $C-V$ hysteresis width is of around $200 \mathrm{mV}$.

Fig. 3(a) shows the $I_{D^{-}} V_{\mathrm{G}}$ transfer characteristics of the $\mathrm{Ge}$ nMOSFET with a NiGe Schottky junction S/D in the linear region at $V_{\mathrm{DS}}=0.1 \mathrm{~V}$ and saturation region at $V_{\mathrm{DS}}=1 \mathrm{~V}$. The schematic cross-sectional view of the device with an $L_{\text {Channel }}$ of $5 \mu \mathrm{m}$ is shown in the inset of Fig. 3(a). A high $I_{\mathrm{ON}} / I_{\mathrm{OFF}}$ ratio of $\sim 10^{5}\left(I_{\mathrm{S}}\right), \sim 8 \times 10^{3}\left(I_{\mathrm{D}}\right)$ at $V_{\mathrm{DS}}=1 \mathrm{~V}$, and a good subthreshold swing of $138 \mathrm{mV} / \mathrm{dec}$ are illustrated. A driving current of $7.3 \mu \mathrm{A} / \mu \mathrm{m}$ at $V_{\mathrm{GS}}-V_{\mathrm{T}}=1.3 \mathrm{~V}$ and a low drain leakage current of $\sim 1 \mathrm{nA} / \mu \mathrm{m}$ at $V_{\mathrm{GS}}-V_{\mathrm{T}}=-0.7 \mathrm{~V}$ were attributed to the thin tunnel barrier for electrons and a high effective $\Phi_{\mathrm{Bp}} \sim 0.57 \mathrm{eV}$ for holes, respectively. A peak mobility $\sim 440 \mathrm{~cm}^{2} / \mathrm{V}$-s was extracted by split $C-V$ (not shown). Recently, numerous articles focusing on the Ge nMOSFETs with the conventional $n^{+} / p$ junction S/D have been published. Fu et al [17] reported a Ge nMOSFET $(\mathrm{S} . \mathrm{S} .=150 \mathrm{mV} / \mathrm{dec})$ with a source current $I_{\mathrm{ON}} / I_{\mathrm{OFF}}$ ratio $\sim 2 \times 10^{4}$ at $V_{\mathrm{D}}=1 \mathrm{~V}$, in which the $I_{\mathrm{ON}}$ was $\sim 3 \mu \mathrm{A} / \mu \mathrm{m}$ at $V_{\mathrm{G}}=1.5 \mathrm{~V}$. Zhang et al [18] reported a $\mathrm{Ge}$ nMOSFET on a (100) wafer with a poor drain current $I_{\mathrm{ON}} / I_{\mathrm{OFF}} \sim 10^{2}$ at $V_{\mathrm{D}}=1 \mathrm{~V}$ and a high drain leakage current of $>10 \mathrm{nA} / \mu \mathrm{m}$ at $V_{\mathrm{G}}=-1 \mathrm{~V}$. Lee et al [19] presented a Ge nMOSFET on a (111) plane with a driving current of $\sim 4 \mu \mathrm{A} / \mu \mathrm{m}$ at $V_{\mathrm{G}}=1.5 \mathrm{~V}$ and $V_{\mathrm{D}}=1 \mathrm{~V}$ and a drain current $I_{\mathrm{ON}} / I_{\mathrm{OFF}}$ ratio of $\sim 2 \times 10^{3}$ even though the equivalent oxide thickness (EOT) was reported in sub-nanometer. Compared with the former results [17]-[19], the Ge nMOSFET with a NiGe Schottky S/D fabricated in this letter exhibited a 


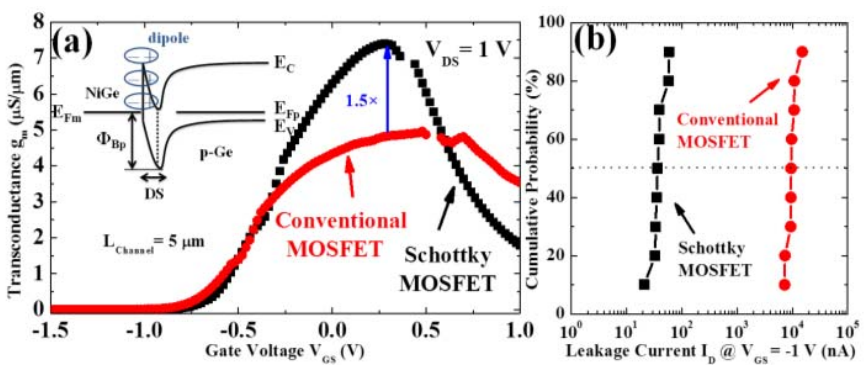

Fig. 4. (a) $g_{\mathrm{m}}$ versus $V_{\mathrm{GS}}$ characteristic of nMOSFET with a Schottky S/D and conventional $\mathrm{S} / \mathrm{D}$ at $V_{\mathrm{DS}}=1 \mathrm{~V}$ and the inset shows the speculative band diagram of $\mathrm{NiGe} / \mathrm{p}-\mathrm{Ge}$ with $\mathrm{DS}$ in thermal equilibrium. (b) Cumulative plot versus drain leakage current of nMOSFETs at $V_{\mathrm{GS}}=-1 \mathrm{~V}$.

superior performance regarding the $I_{\mathrm{ON}} / I_{\mathrm{OFF}}$ ratio, and the SS. Fig. 3(b) shows the $I_{\mathrm{D}}-V_{\mathrm{DS}}$ output characteristics of the Ge nMOSFET; the saturation current of the device was $4 \mu \mathrm{A} / \mu \mathrm{m}$ at $V_{\mathrm{DS}}$ and $V_{\mathrm{GS}}-V_{\mathrm{T}}=1 \mathrm{~V}$. In previous studies [20], [21], numerous methods have been proposed for improving the S/D series resistance of nMOSFETs to enhance the driving current; however, the devices with a Schottky junction S/D shows the highest output current [20], [21]. In addition, a nMOSFET with a conventional $\mathrm{n}^{+} / \mathrm{p}$ junction $\mathrm{S} / \mathrm{D}$ and $\mathrm{NiGe}$ contact was fabricated for comparison. The transconductance $\left(g_{\mathrm{m}}\right)$ versus gate voltages is shown in Fig. 4(a). The peak $g_{\mathrm{m}}$ value of the Schottky MOSFET was around 1.5 times higher than that of the conventional MOSFET at $V_{\mathrm{DS}}=1 \mathrm{~V}$. Because the phosphorus dopants were segregated near the $\mathrm{NiGe/p-Ge} \mathrm{interface,} \mathrm{the} \mathrm{combined} \mathrm{effects} \mathrm{of}$ the surface dipoles and the band bending were determined to modulate the electron tunneling barrier; the speculated band diagram in thermal equilibrium is shown in the inset of Fig. 4(a). On the other hand, the hole effective $\mathrm{SBH}$ $(\sim 0.57 \mathrm{eV})$ can also be modulated by the dopants, which leads to the considerably suppressed hole current (leakage current) in the off state. Fig. 4(b) shows the cumulative plot of the drain leakage current at $V_{\mathrm{GS}}=-1 \mathrm{~V}$; the leakage current of the SBH MOSFET is at least two orders of magnitude lower than that of the conventional MOSFET. The narrow distribution of the low leakage current indicates that the DS process is extremely stable. As a result, a high performance Ge nMOSFET with NiGe S/D was demonstrated; the DS technique was employed to alleviate the FLP effect and an ohmic contact was successfully changed to a Schottky contact, which resulted in a low leakage current.

\section{CONCLUSION}

In conclusion, a high performance Ge nMOSFET with a NiGe Schottky junction S/D was fabricated using the DS technique. The device exhibited a high $I_{\mathrm{ON}} / I_{\mathrm{OFF}}$ ratio of $\sim 10^{5}$ (source current), and a SS of $138 \mathrm{mV} / \mathrm{dec}$. The high driving current of $7.3 \mu \mathrm{A} / \mu \mathrm{m}$ at $V_{\mathrm{GS}}-V_{\mathrm{T}}=1.3 \mathrm{~V}$ and low drain leakage current of $\sim 1 \mathrm{nA} / \mu \mathrm{m}$ at $V_{\mathrm{GS}}-V_{\mathrm{T}}=-0.7 \mathrm{~V}$ were attributed to the thin tunnel barrier for electrons and the high effective SBH for holes, respectively. Moreover, the study shows that the performance of the Ge nMOSFET with a NiGe Schottky S/D is superior to that of a conventional device. Finally, the results indicate that this high performance Ge nMOSFET with a NiGe Schottky S/D can be applied in Ge CMOS realization.

\section{REFERENCES}

[1] G.-L. Luo, S.-C. Huang, and C.-T. Chung, "A comprehensive study of $\mathrm{Ge}_{1-\mathrm{X}} \mathrm{Si}_{\mathrm{X}}$ on $\mathrm{Ge}$ for the Ge nMOSFETs with tensile stress, shallow junctions and reduced leakage," in Proc. IEEE IEDM, Dec. 2009, pp. 689-692.

[2] J.-H. Park, M. Tada, D. Kuzum, et al., "Low temperature $\left(\leq 380{ }^{\circ} \mathrm{C}\right)$ and high performance Ge CMOS technology with novel source/drain by metal-induced dopants activation and high-k/metal gate stack for monolithic 3D integration," in Proc. IEEE IEDM, Dec. 2008, pp. 389-392.

[3] Y. Nakakita, R. Nakane, T. Sasada, et al., "Interface-controlled self-align source/drain Ge pMOSFETs using thermally-oxidized $\mathrm{GeO}_{2}$ interfacial layers," in Proc. IEEE IEDM, Dec. 2008, pp. 877-880.

[4] T. Yamamoto, Y. Yamashita, M. Harada, et al., "High performance $60 \mathrm{~nm}$ gate length germanium p-MOSFETs with $\mathrm{Ni}$ germanide metal source/drain," in Proc. IEEE IEDM, Dec. 2007, pp. 1041-1043.

[5] C. O. Chui and K. C. Saraswat, Germanium-Based Technologies: From Materials to Devices. Amsterdam, The Netherlands: Elsevier, 2007.

[6] F. A. Trumbore, "Solid solubilities of impurity elements in germanium and silicon," Bell Syst. Tech. J., vol. 39, no. 1, pp. 205-233, Jan. 1960.

[7] C. O. Chui, K. Gopalakrishnan, P. B. Griffin, et al., "Activation and diffusion studies of ion-implanted $\mathrm{p}$ and $\mathrm{n}$ dopants in germanium," Appl. Phys. Lett., vol. 83, no. 16, pp. 3275-3277, Oct. 2003.

[8] C. O. Chui, L. Kulig, J. Moran, et al., "Germanium n-type shallow junction activation dependences," Appl. Phys. Lett., vol. 87, no. 9, pp. 091909-1-091909-3, Aug. 2005.

[9] C. H. Poon, L. S. Tan, B. J. Cho, et al., "Dopant loss mechanism in $\mathrm{n}^{+} / \mathrm{p}$ germanium junctions during rapid thermal annealing," J. Electrochem. Soc., vol. 152, no. 12, pp. G895-G899, 2005.

[10] M. Mueller, Q. T. Zhao, C. Urban, et al., "Schottky-barrier height tuning of NiGe/n-Ge contacts using As and $\mathrm{P}$ segregation," Mater. Sci. Eng. B, vols. 154-155, pp. 168-171, Dec. 2008.

[11] B. Yang, J.-Y. J. Lin, S. Gupta, et al., "Low-contact-resistivity nickel germanide contacts on $\mathrm{n}+\mathrm{Ge}$ with phosphorus/antimony co-doping and Schottky barrier height lowering," in Proc. ISTDM, Jun. 2012, pp. 1-2.

[12] Z. Li, X. An, M. Li, et al., "Low electron Schottky barrier height of $\mathrm{NiGe} / \mathrm{Ge}$ achieved by ion implantation after germanidation technique," IEEE Electron Device Lett., vol. 33, no. 12, pp. 1687-1689, Dec. 2012.

[13] M. Koike, Y. Kamimuta, and T. Tezuka, "Modulation of NiGe/Ge Schottky barrier height by S and P co-introduction," Appl. Phys. Lett., vol. 102, no. 3, pp. 032108-1-032108-4, Jan. 2013

[14] T. Nishimura, S. Sakata, K. Nagashio, et al., "Low temperature phosphorus activation in germanium through nickel germanidation for shallow $\mathrm{n}^{+} / \mathrm{p}$ junction," Appl. Phys. Express, vol. 2, pp. 021202-1-021202-3, Jan. 2009.

[15] D. K. Schroder, Semiconductor Material and Device Characterization. Hoboken, NJ, USA: Wiley, 2006.

[16] E. H. Nicollian and J. R. Brews, MOS (Metal Oxide Semiconductor) Physics and Technology. New York, NY, USA: Wiley, 1982.

[17] Y.-C. Fu, W. Hsu, Y.-T. Chen, et al., "High mobility high on/off ratio $C-V$ dispersion-free Ge n-MOSFETs and their strain response," in Proc. IEEE IEDM, Dec. 2010, pp. 432-435.

[18] R. Zhang, J.-C. Lin, X. Yu, et al., "Examination of physical origins limiting effective mobility of Ge MOSFETs and the improvement by atomic deuterium annealing," in Proc. Symp. VLSI Technol., Jun. 2013, pp. T26-T27.

[19] C. H. Lee, C. Lu, T. Tabata, et al., "Enhancement of high-Ns electron mobility in sub-nm EOT Ge n-MOSFETs," in Proc. Symp. VLSI Technol., Jun. 2013, pp. T28-T29.

[20] Y. Kamata, K. Ikeda, Y. Kamimuta, et al., "High-k/Ge p- \& n-MISFETs with strontium germanide interlayer for EOT scalable CMIS application,” in Proc. Symp. VLSI Technol., Jun. 2010, pp. 211-212.

[21] H.-Y. Yu, M. Kobayashi, J.-H. Park, et al., "Novel germanium n-MOSFETs with raised source/drain on selectively grown $\mathrm{Ge}$ on $\mathrm{Si}$ for monolithic integration,' IEEE Electron Device Lett., vol. 32, no. 4, pp. 446-448, Apr. 2011. 